\title{
Canadian Urological Association guideline for the management of bladder and bowel dysfunction in children
}

Kourosh Afshar, MD, MHSc, FRCSC ${ }^{1}$; Joana Dos Santos, MD, MHSc, FRCPC ${ }^{2}$; Anne-Sophie Blais, MD, FRCSC ${ }^{3}$; Darcie Kiddoo, MD, FRCSC ${ }^{4}$; Nafisa Dharamsi, MD, FRCSC ${ }^{5}$; Mannan Wang, $\mathrm{MD}^{1}$; Maryam Noparast, $\mathrm{MD}^{1}$

${ }^{1}$ Department of Urologic Sciences, University of British Columbia, Vancouver, BC, Canada; ${ }^{2}$ Division of Urology, SickKids, Toronto, ON, Canada; ${ }^{3} \mathrm{CHU}$ de Quebec, Université Laval, Quebec City, QC, Canada; ${ }^{4}$ Department of Surgery, University of Alberta, Edmonton, AB, Canada; ${ }^{5}$ Department of Surgery, Section of Urology, University of Manitoba, Winnipeg, MB, Canada

Cite as: Afshar K, Dos Santos J, Blasi A-S, et al. Canadian Urological Association guideline for the management of bladder and bowel dysfunction in children. Can Urol Assoc J 2020 September 28; Epub ahead of print. http://dx.doi.org/10.5489/cuaj.6975

Published online September 28, 2020

$* * *$

\section{Introduction}

Bladder and bowel dysfunction (BBD) is one of the most common reasons for referral to pediatric urology clinics, responsible for up to $40 \%$ of clinic consults (1). BBD describes a constellation of symptoms related to voiding and defecation without a neurogenic or anatomic cause. The association of bowel and bladder symptoms is well described (2). The lower urinary tract symptoms (LUTS) include storage type such as urgency, frequency and urge incontinence or voiding type such as hesitancy, slow urinary flow and intermittency. Gastrointestinal symptoms include constipation and encopresis.

The term BBD is applied to a heterogeneous group of clinical presentations. Some children present primarily with frequency, urgency with or without incontinence, others postpone their urination and do not empty their bladder. In an effort to standardize the terminology related to BBD, its subtypes and symptoms, International Children's Continence Society (ICCS) has published a classification, which is gaining more acceptance in pediatric urology literature(3). We have strived to align this manuscript with this classification.

BBD is a known risk factor for urinary tract infection (UTI) and vesicoureteral reflux (VUR) (4). Many studies have shown the importance of BBD management in prevention of UTIs and treatment of VUR (5). BBD is associated with reduced quality of life and significant psychosocial burden for children and families (6). It is not uncommon for children with BBD to be stigmatized and bullied. Mood disorders and anxiety are also seen in these children (7).

BBD is a clinical construct. Many different validated questionnaires, such as the DVSS and Vancouver symptom scores, have been designed in an attempt to standardize the diagnosis, 
classify the type, and evaluate the severity of this complex clinical diagnosis. These instruments have also been used to follow clinical response to treatment $(8,9,10)$.

The treatment of bowel dysfunction is an essential part of the overall management and should not be overlooked. The scope of the current guidelines is limited to the management of the lower urinary tract.

The purpose of these guidelines is to identify the best available evidence regarding the management of BBD in children, assess the level and quality of the evidence and generate recommendations for clinicians.

\section{Methods}

A systematic approach to literature search was used to identify the relevant studies. A comprehensive literature search strategy was written by experienced librarian. Embase, Medline, google scholar, Cochrane library for randomized control trials and clinical trials.gov were searched. We limited our search to randomized (or quasi-randomized) controlled trials (RCT) that compared at least one active treatment modality with another, placebo or observation. We only included studies with participants up to 18 years of age. Outcomes of interest included patient reported outcomes such as change in symptoms, change in scores of validated questionnaires or uroflowmetric parameters and the incidence of UTI. Quality of life and adverse events have also been included as outcomes.

Cochrane collaborative methodology was used to assess the titles, abstracts and articles for inclusion and exclusion, data extraction, assessment of bias and synthesis. Each step was independently completed by at least 2 investigators. Results were reviewed by the senior author who acted as the tiebreaker in the case of disagreement. Quality of each study was evaluated based on Cochrane collaborative criteria (11). Whenever possible, data were pooled from different studies using a random effect model metanalysis.

Finally, the recommendations were generated according to Grading of Recommendations Assessment, Development, and Evaluation (GRADE) methodology, using GRADE Pro software (12).

\section{Results}

We searched the literature up to November $4^{\text {th }}, 2019$. Our literature search yielded 1069 titles of which 179 studies were included for full review based on our a priori inclusion/exclusion criteria. We are presenting the results of our search based on the interventions. We faced a tremendous challenge when assessing these studies. Apart from low quality of evidence in general, the literature is plagued with non-standardized use of clinical terms, incomplete reporting of results and focus on clinically non important outcomes. Many studies used the same nomenclature for management strategies with a vastly different protocol. This has prevented the pooling of results from many studies. 


\section{Treatments}

Bladder retraining/urotherapy

We were not able to identify a study comparing urotherapy with observation only. This is understandable given the simplicity of urotherapy and the lack of adverse events. Most studies evaluated some variations of urotherapy.

The other important note is that protocols of urotherapy varied widely in terms of method of delivery, contents, length and frequency of treatment, and inclusion of additional interventions, such as behavioral or cognitive therapy. Nevertheless, all regimens included timed voiding, fluid intake and dietary strategies, and management of constipation.

1. One RCT comparing standard urotherapy with and without timer for scheduled voiding in children with urge incontinence, found a significant improvement in median number of wet days/week in favor of using timer ( at $12^{\text {th }}$ week follow-up median number of wet days/week were 2 and 5 in timer vs standard group). Complete response was seen in $30 \%$ of children in timer group vs. none in the standard urotherapy group (Hagstroem 2010). GRADE Level: Moderate.

2. One RCT compared combination of instructional home video and behavioral therapy to standard behavioral therapy in children with dysfunctional voiding and recurrent UTIs (Klijn 2006). At 12 months follow up there was no difference between the 2 groups in terms of resolution of incontinence or recurrence of UTI. GRADE level: Low

3. In another RCT, 150 children with BBD diagnosed using the Vancouver symptom score, were randomized to receive standard urotherapy vs an instructional video. This study had a non-inferiority design. The authors did not find the video inferior to the standard management in reducing the symptom score at a mean follow up of 3 months (Braga 2017). GRADE Level: Moderate

4. Group urotherapy (one-hour session) and individual urotherapy (15 minute session) are equally effective in reducing symptom score and improving disease specific quality of life in children with BBD, at a median follow up of 14 weeks. (Brownrigg 2015). GRADE Level: Moderate.

\section{Biofeedback}

1. In a RCT of 94 children with dysfunctional voiding and high post-void residual, addition of biofeedback to standard urotherapy is not associated with improvement of uroflow- metric parameters such as average maximum flow rate at six months of follow up (Kibar 2010). GRADE Level: Moderate. Nevertheless, post void residual decreased $20 \mathrm{cc}$ in average in children who received biofeedback. GRADE Level: Low.

2. In 40 children with dysfunctional voiding animated biofeedback and non-animated biofeedback are no different in reducing symptom scores or improving uroflowmetric 
parameters such as maximum flow rate, post-void residuals and voided volumes (Oktar 2017). GRADE Level Low.

3. In 50 children with underactive bladder addition of biofeedback to standard urotherapy was associated with significant reduction of perineal EMG activity during voiding at 6 and 12 months (OR 0.25 95\% CI 0.07-0.83), as well as likelihood of abnormal voiding pattern (OR 0.17 95\% CI 0.05-0.53) ) GRADE Level: High. Biofeedback also resulted in an average of 2 more voids per day compared to standard treatment. There was a significant reduction in post void residual at 12 months of follow up (mean difference between groups $34.5 \mathrm{cc}$ ) GRADE Level: High.

4. Children in biofeedback group were twice as likely to be dry during the day at 12 months follow up (OR2.1 95\% CI 1.36-2.84). (Ladi-Seyedian 2015) GRADE level: High.

5. Metanalysis of 3 randomized controlled trials in 125 children with BBD (Klijn 2006, Vasconcelos 2006, Kajbafzadeh 2011) did not show any difference between biofeedback (with or without pelvic floor exercise) and standard treatment in resolving daytime incontinence (Fazeli 2015). GRADE Level Low

6. Metanalysis of 4 randomized controlled trials in 163 children with BBD (Klijn 2006, Vasconcelos 2006, Kibar 2010, Kajbafzadeh 2011) did not show any difference between biofeedback and standard treatment in resolving daytime incontinence (Fazeli 2015). GRADE Level: Low

7. Metanalysis of 4 randomized controlled trials did not show any difference between biofeedback and standard treatment in reduction of incidence of UTI (Fazeli 2015). GRADE Level: Very Low

\section{Pelvic floor physiotherapy}

1. Metanalysis of 2 randomized controlled studies (Ladi-Seyedian 2014, Zivkovic 2010) showed that the addition of pelvic floor exercise to the standard treatment in children with dysfunctional voiding is associated with lower likelihood of daytime incontinence at 12 months. (odds ratio 0.14, 95\%CI: 0.05-0.38. Absolute effect 295 fewer per 1000). GRADE level: Moderate.

2. There was no significant difference in the likelihood of UTI or resolution of enuresis. GRADE Level Low and Moderate, respectively.

\section{Neuromodulation}

\section{Parasacral transcutaneous electrical nerve stimulation (TENS)}

1. One RCT did not show any benefit in adding parasacral TENS to standard treatment in improving uroflowmetric variables or clinical outcomes such as frequency in 62 children with overactive bladder (Sillen 2014). GRADE Level: Low 
2. One RCT in 43 children with urge incontinence showed that the combination of parasacral TENS and Oybutynin is associated on average with 2 more dry days per week (MD 2.28 95\% CI 0.5-4.06) compared to TENS and placebo (GRADE level: Low). In the same study no participants in the placebo group achieved total continence as opposed to $36 \%$ in the oxybutynin group (Borch 2017). GRADE Level: Low

3. The same study also compared adding TENS or Sham TENS to oxybutynin in 45 patients. There was no difference between the active and sham groups in terms of resolution of incontinence at 10-week follow up. GRADE Level: Low

4. Another small RCT on 27 children with refractory urge incontinence with only 4 weeks follow up, S2-3 TENS was associated with 3 fewer day per week of incontinence compared to sham TENS. Study groups were not similar at the baseline (Hagstroem 2009). GRADE Level: Low

5. One RCT compared the combination of parasacral TENS and placebo to Oxybutynin and sham TENS. Twenty-eight children with overactive bladder were recruited (Quintliano 2014). At 3-month there was no significant difference in the 2 group in regards to mean change in voiding frequency. Change in symptom score, maximum voided volume and mean voided volumes were similar in the 2 groups. GRADE Level: Low

6. One RCT including only 16 children with overactive bladder compared the effect of parasacral TENS to sham TENS. All patients received urotherapy as well. There was no difference in volumetric variables at 2 months follow up. Fewer patients had urgency at 2 months follow up (RR $3.7595 \%$ CI 1.01 to 13.8). GRADE Level Very Low. They also reported subjective improvement based on a visual analog scale(VAS) with no confirmed validity of the measurement which makes interpretation unfeasible. (DePaula 2017)

Posterior tibial transcutaneous electrical stimulation (PTTENS)

1. A small RCT compared PTTENS with sham treatment in 20 patients with refractory overactive bladder. The study showed increase in mean voided volumes (MD change $84.2 \mathrm{cc}$ ) but no change in bladder capacity, post void residual and clinical variables as measured by a non validated symptom score (Boudaoud 2015). Grade Level: Low

2. In one study of 37 children with refractory overactive bladder PTTENS was compared to sham TENS. The authors reported subjective improvement with no quantifiable measures (Patidar 2015). It showed a favorable response at 3 months towards TENS as 14/21 had full response as opposed to 0/16 in the control group. GRADE Level Very Low. 


\section{Inferential pelvic transcutaneous electrical nerve stimulation}

1. One study has compared this treatment to standard urotherapy in 36 children with underactive bladder (Kajbafzadeh 2016). At 12 month follow up the results were favoring TENS: number of voiding voids per day were higher, on average 1.6 times (GRADE Level: Very Low), bladder capacity was lower by $117 \mathrm{~mL}(95 \% \mathrm{CI}$ 46-188 mL), (GARDE Level: Low), voiding time was 18 second shorter ( $95 \% \mathrm{CI}$ 8-27 sec.) (GARDE Level: Low) and PVR was smaller by approximately $10 \%$ of bladder capacity (GRADE level: High). Urinary flow rate was similar in the 2 groups. (GRADE Level: High) Number of voids per day did not change significantly at 12 months. (GRADE Level: High)

\section{Pharmacotherapy}

1. Solifenacin: A randomized placebo controlled trial comparing Solifenacin and placebo in 189 children and adolescents showed that mean voided volume per micturition was $12.1 \mathrm{~mL}$ higher ( $95 \% \mathrm{CI} 0.2-24)$ in 148 children (5-12 years old) who received Solifenacin (Newgreen 2017) GRADE Level Low. The magnitude of change was not clinically significant. The maximum voided volume per micturition was higher by an average of $31.9 \mathrm{~mL}$ (95\% CI 4.3-59.5). GRADE Level Low

2. The study did not show any other significant effect on more clinically important outcomes such as number of voids or wet days. Due to low number of adolescents (41) the study did not reach any conclusion in this group.

3. Propiverine: One RCT (Marschall-Kejrel 2009) compared Propiverine to placebo in 164 children 5-17 year of age with overactive bladder. Endpoints were assessed at 8 weeks. Efficacy was compared in the 2 groups. Mean voided volume was higher by an average of $26.3 \mathrm{ml}$. in the treatment group (95\% CI $18.0-34.6 \mathrm{~mL})$ GRADE level: Moderate. Treatment was associated with a modest reduction in daily voiding frequency ( 0.8 fewer voids per day $95 \%$ CI $0.11-1.5$ ) over placebo. GRADE Level: Moderate.

4. Two randomized placebo-controlled studies were reported in a single publication by Nijman et al. comparing extended release tolterodine with placebo in children with urge incontinence (Nijman 2005). The baseline characteristics of participants were slightly different. Outcomes were evaluated at 12 weeks. The effect of drug was more obvious in patients with more than 6 voids per day. Pooling the results of the two studies showed a modest 1.4 fewer (95\% CI 0.13-2.71) urge incontinence episodes per week. GRADE Level: moderate

5. A randomized placebo-controlled study in 42 children presenting with daytime incontinence showed that terodiline is associated with a modest effect of one fewer (95\% CI 0.17-1.83) wet episode per day. GRADE Level: low. There was no effect 
on nighttime enuresis. Inclusion criteria were broad and vague preventing pooling data with other studies (Elmer 1988).

6. In a subgroup of a complex RCT (van Gool 2014), including 63 children with overactive bladder, divided into 2 groups, adding oxybutynin or placebo to cognitive therapy was not associated with a different cure rate at 12 months follow up (43\% vs 33\%, OR $1.1895 \%$ CI 0.43 to 3.21). GRADE Level: Low.

\section{Adverse events}

Nijman et al. did not show any difference in adverse events such as headache, gastrointestinal issues (diarrhea/nausea/vomiting) or UTI, in children receiving tolterodine vs placebo (GRADE Level: Moderate). The study did not report the incidence of constipation. One percent of patients had serious adverse events, but none of them have been attributed to the treatment (Nijman 2005). Study by Marschall did not show an increase in adverse events in children treated with propiverine (GRADE Level: Moderate). They reported a $\%$ incidence of constipation in the treatment group.

\section{Discussion}

The primary objective of these guidelines is to provide recommendations for urological management of children with bladder and bowel dysfunction (BBD) based on best available evidence. These guidelines do not include the primary treatment of gastrointestinal symptoms such as constipation, although we recognize this is an important part of the comprehensive management. The guidelines are based on the findings of randomized controlled trials. To maintain the highest quality of recommendations we excluded observational studies.

The development of the guidelines followed the CUA recommended methods to identify, assess and synthesize the best available evidence. The steps in this endeavour include a systematic search of the literature using a comprehensive search strategy written by experienced medical librarian, review of the titles abstracts, data extraction and assessment of bias form included studies by 2 investigators in an independent fashion. Whenever possible the results of the studies were pooled using meta-analytic methods. We used the GRADE system to assess the evidence and develop recommendations. Although a full explanation of the Grading of Recommendation Assessment Development and Evaluation (GRADE) methodology is outside the scope of this manuscript, a short description may be helpful. This methodology is used to reduce the confusion arising from multiple systems for grading evidence and provide the clinicians the level of certainty for each recommendation (12). The evidence for each study or pooled results of several studies are judged based on risk of bias, imprecision of the effect size, inconsistency, indirectness of findings in terms of sample or outcomes and when applicable publication bias. Once the assessment is completed the rating below is applied to the recommendations:

\section{Very low The true effect is probably markedly different from the estimated effect}

Low

The true effect might be markedly different from the estimated effect 


\section{Moderate The authors believe that the true effect is probably close to the estimated effect}

\section{High The authors have a lot of confidence that the true effect is similar to the estimated effect}

During this review we encountered several prevalent challenges that made assessment of the quality, estimation of the effect size and pooling of the data very onerous. Unclear or suboptimal randomization (selection bias), non-blinded studies (performance bias) and incomplete reporting (reporting bias) are among the most common issues affecting the quality of the evidence. The inclusion-exclusion criteria for recruiting patients have been vastly different among the studies. This has been magnified by the lack of a common terminology for the conditions under the rubric of BBD that has been partly remediated by the efforts of International Children's Continence Society (2). Studies used different interventions although they may have been named similarly. For example, the words urotherapy or bladder retraining encompass a wide variety of regimen that includes patient education, timed voiding, fluid management, behavioral modification techniques and many more interventions with different time frame and application methods. In addition, many studies focused on surrogate outcomes that are not clinically important such as uroflowmetric parameters (mean voided volumes, maximum voided volumes etc..) and either ignored more clinically relevant outcomes (incontinence, resolution of symptoms etc..) reported by the patients or could not reach a conclusion due to small sample size. Follow up lengths were also very variable.

This heterogeneity in population, interventions and outcomes prohibited us from pooling the data and performing meta-analysis in many occasions.

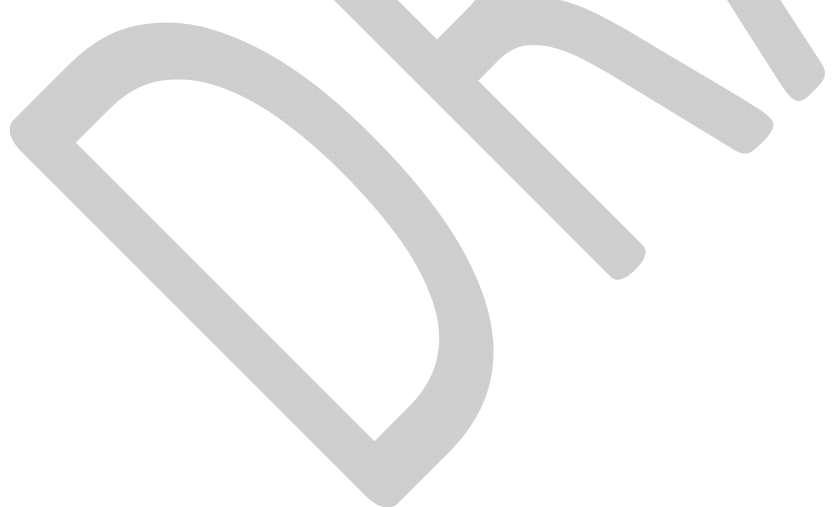




\section{Recommendations}

1. Urotherapy/bladder retraining with timer to assist scheduled voiding is recommended over the same treatment without timer. (GRADE Level: Moderate)

2. Face to face (group or individual) bladder retraining and video instructions are equally effective. (GRADE Level: Low to Moderate)

3. In children with underactive bladder addition of biofeedback to standard urotherapy is beneficial. (GRADE Level: High)

4. Biofeedback in children with other types of BBD is not associated with improved outcomes. (GRADE Level Low)

5. Addition of pelvic floor muscle physiotherapy to urotherapy has a beneficial effect on resolution of daytime incontinence in children with dysfunctional voiding. (GRADE Level: Moderate)

6. Para-sacral Transcutaneous Electrical Nerve Stimulation (PS-TENS)

a. No evidence to support PS-TENS as an effective adjunct to urotherapy or oxybutynin for overactive bladder. (GRADE Level: Low)

b. TENS may be useful in management of refractory urge incontinence in short term, by reducing the number of wet days. (GRADE Level: Low)

7. Inferential Transcutaneous Electrical Nerve Stimulation may increase the voiding frequency and uroflowmetric parameters (e.g PVR) in short term, however, there is no evidence it is more effective than urotherapy in long term management of children with underactive bladder. (GRADE Level:Ligh)

8. Anticholinergics:

a. Solifenacin: May increase the mean and maximum voided volumes in children with overactive bladder, but it may not be different from placebo in improving incontinence or number of daily voids. (off label use) (GRADE Level: Low).

b. Propiverine: May increase mean voided volumes and modestly reduce daily frequency compared to placebo in children with overactive bladder. (GRADE Level: Moderate)

c. Tolterodine extended release may result in a small decrease in urge incontinence in children with overactive bladder (average 1.4 incontinence episodes per week) when compared to placebo. (off label use) (GRADE Level: Moderate)

d. We found no evidence of difference between oxybutynin and cognitive therapy in cure rate of incontinence in children with overactive bladder. (GRADE Level: Low) 


\section{References}

1. Santos JD, Lopes RI, Koyle MA. Bladder and bowel dysfunction in children: An update on the diagnosis and treatment of a common, but underdiagnosed pediatric problem. Can Urol Assoc J. 2017;11(1-2Suppl1):S64-S72. doi:10.5489/cuaj.4411

2. S. Yazbeck, E. Schick, S. O'Regan: Relevance of constipation to enuresis, urinary tract infection and reflux Eur Urol, 13 (1987), pp. 318-321

3. Austin $\mathrm{P}$, Bauer $\mathrm{S}, \mathrm{Neveus} \mathrm{T}$. The standardization of terminology of lower urinary tract function in children and adolescents: update report from the Standardization Committee of the International Children's Continence Society. J Urol 2014;191:1863-5.

4. Elder JS, Diaz M. Vesicoureteral reflux--the role of bladder and bowel dysfunction. Nat Rev Urol. 2013;10(11):640-648. doi:10.1038/nrurol.2013.221

5. Edwards A, Peters CA. Managing vesicoureteral reflux in children: making sense of all the data. F1000Res. 2019;8:F1000 Faculty Rev-29. Published 2019 Jan 8. doi:10.12688/f1000research.16534.1

6. Chase J, Bower W, Gibb S, Schaeffer A, von Gontard A. Diagnostic scores, questionnaires, quality of life, and outcome measures in pediatric continence: A review of available tools from the International Children's Continence Society. J Pediatr Urol. 2018;14(2):98-107. doi:10.1016/j.jpurol.2017.12.003

7. Morgan KE, Leroy SV, Corbett ST, Shepard JA. Complementary and Integrative Management of Pediatric Lower Urinary Tract Dysfunction Implemented within an Interprofessional Clinic. Children (Basel). 2019;6(8):88. Published 2019 Jul 30. doi:10.3390/children6080088

8. Farhat $\mathrm{W}$, et al. The dysfunctional voiding scoring system:quantitative standardization of dysfunctional voiding symptoms in children. J Urol 2000;164(3 Pt 2):1011e5.

9. Afshar K, et al. Development of a symptom score for dysfunctional elimination syndrome. J Urol 2009;182(4 Suppl): 1939e43.

10. Jiang R, Kelly MS, Routh JC. Assessment of pediatric bowel and bladder dysfunction: a critical appraisal of the literature. J Pediatr Urol. 2018;14(6):494-501. doi:10.1016/j.jpurol.2018.08.010

11. https://training.cochrane.org/handbook/current accessed May 19, 2020

12. https://www.gradeworkinggroup.org/ 